\title{
SINGULAR VALUE ANALYSIS OF SPACE-TIME EQUALIZATION IN THE GSM MOBILE SYSTEM
}

\author{
Alle-Jan van der Veen ${ }^{\dagger *}$ and A. Paulraj* \\ †Delft University of Technology, Dept. Electrical Engineering/DIMES, 2628 CD Delft, The Netherlands \\ * Stanford University, Information Systems Laboratory, Stanford, CA 94305
}

Singular value analysis of a GMSK-modulated signal such as employed by GSM reveals that it admits a reasonably accurate linear model, enabling the use of linear space-time equalizers to retrieve the data symbols. The analysis also shows that one antenna does not provide sufficient resolution to allow estimation of the channel length, so that the performance of equalizers is limited. An algorithm is proposed for the blind space-time equalization and separation of multiple co-channel GMSK signals, based on their fixed symbol rate, finite alphabet and constant modulus properties.

\section{INTRODUCTION}

Under conditions, multi-user wireless channels transmitting finite alphabet symbols can be identified blindly. The algorithms in [1-3] take observed data from multiple oversampled antennas, and assume linear modulation and linear FIR channels. In this paper, we apply the algorithm from [3] to GMSK phase modulated signals received by base stations in the GSM mobile system. Since this modulation scheme is nonlinear, we first investigate whether such signals are aptly described by a linear model. This turns out to be the case. Subsequently, we look at the transmission of GMSK signals over the ETSI-specified hilly terrain channel model [4]. Based on the singular values of the data matrix, it is seen that the information from only one antenna does not provide sufficient resolution to even identify the channel length, implying that exact equalization is not possible, and that equalizers will be sensitive to noise even if they are based on training sequences. This resolution problem is basically due to the bandlimited nature of the GMSK signal, and agrees with a recently developed theory [5].

Finally, we look at blind identification algorithms to separate and equalize GMSK signals of multiple co-channel users transmitted over FIR channels. A number of properties of the signal can be used:

1. fixed symbol rate of the signals, which allows to obtain independent linear combinations of the same symbols by using oversampling and/or multiple antennas (assuming linear modulation),

2. the constant modulus $(\mathrm{CM})$ of the signals,

3 . the finite alphabet (FA) of the symbols.

The algorithms proposed in [1-3] use only properties 1 and 3. Simulations on GMSK signals indicate that they are easily confused by ambiguities in the symbol constellation (caused by the nonlinear modulation scheme), and are sensitive to parameter settings because channel lengths are ill-defined and perhaps unobservable. The first problem is solved by a certain transformation to a reduced symbol constellation. At this point, the CM property coincides with the FA property. By looking at the singular values of a "CM condition matrix", we show that the CM property is strongly present and provides the means for a reliable equalization and separation. The resulting algorithm is similar to that of [2,3], but with the FA step replaced by a variant of the recently derived "analytic CM algorithm" [6]. The FA property is subsequently used for optimization of the estimates.

\section{LINEAR DATA MODEL}

To describe the FIR-MIMO (multi-input multi-output) scenario, consider the linear data model as detailed in [2]. Assuming $M$ antennas, $P$ times oversampling, and an equalizer length of $m$ symbols, the data vectors $\mathbf{x}_{k} \in \mathbb{C}^{M P}$ received at the antenna array during $N$ symbol periods are collected in a block-Hankel matrix $\mathcal{X}$,

$$
\mathcal{X}=\left[\begin{array}{llll}
\mathbf{x}_{0} & \mathbf{x}_{1} & \because & \mathbf{x}_{N-m} \\
\mathbf{x}_{1} & \mathbf{x}_{2} & \because & \therefore \\
\because & \because & \because & \mathbf{x}_{N-2} \\
\mathbf{x}_{m-1} & \because & \mathbf{x}_{N-2} & \mathbf{x}_{N-1}
\end{array}\right] \quad: m M P \times(N-m+1) .
$$

With $d$ users and a maximum channel length of $L$ symbols per channel, $\mathcal{X}$ has a factorization [2]

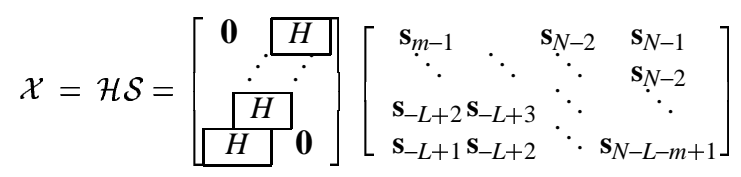

$\mathcal{H}: m M P \times d(L+m-1)$ : block-Hankel,

$\mathcal{S}: d(L+m-1) \times(N-m+1)$ : block-Toeplitz, finite alphabet.

$H$ contains the impulse response of the channel, convolved with the modulating pulse shape function; $\mathbf{s}_{k}$ is a $d \times 1$ vector containing the symbols transmitted by the $d$ users in the $k$-th interval. If $m M P$ is large enough and $\mathcal{H}$ has full column rank, then $\mathcal{X}$ is rank deficient and is expected to have rank

$$
d_{\mathcal{X}}=d(L+m-1) .
$$

The blind FIR-MIMO identification problem may be stated as a matrix factorization problem: given $\mathcal{X}$, find factors $\mathcal{H}$ and $\mathcal{S}$ with the indicated structure. Algorithms to solve this problem under more or less ideal circumstances were proposed in [1-3], and use two properties: (1) the Toeplitz structure of $\mathcal{S}$, which is exploited by subspace intersections on the row span of $\mathcal{X}$ and shifts of this space; (2) the finite alphabet property of the entries of $\mathcal{S}$, which is exploited by alternating projections of candidate signals onto this alphabet and the residual row span after subspace intersections [3]. With only one signal present $(d=1)$, the first property by itself is already sufficient for estimating $\mathcal{S}$. With more than one signal, the second property is necessary for separation. However, even if only one signal is present, use of the second property improves the accuracy and is essential in cases where channel lengths are not welldefined.

In this paper, we are in particular interested in the singular value analysis of $\mathcal{X}$. The singular values give information on the rank of $\mathcal{X}$, which allows the determination of both the number of signals $d$ and the channel lengths $L$. With prior knowledge, they enable to judge whether the number of antennas and amount of oversampling provides sufficient resolution to be able to equalize the channel using linear equalizers. This is relevant also for non-blind algorithms that use training sequences.

IEEE ICASSP'96, Atlanta (GA), May 1996. 

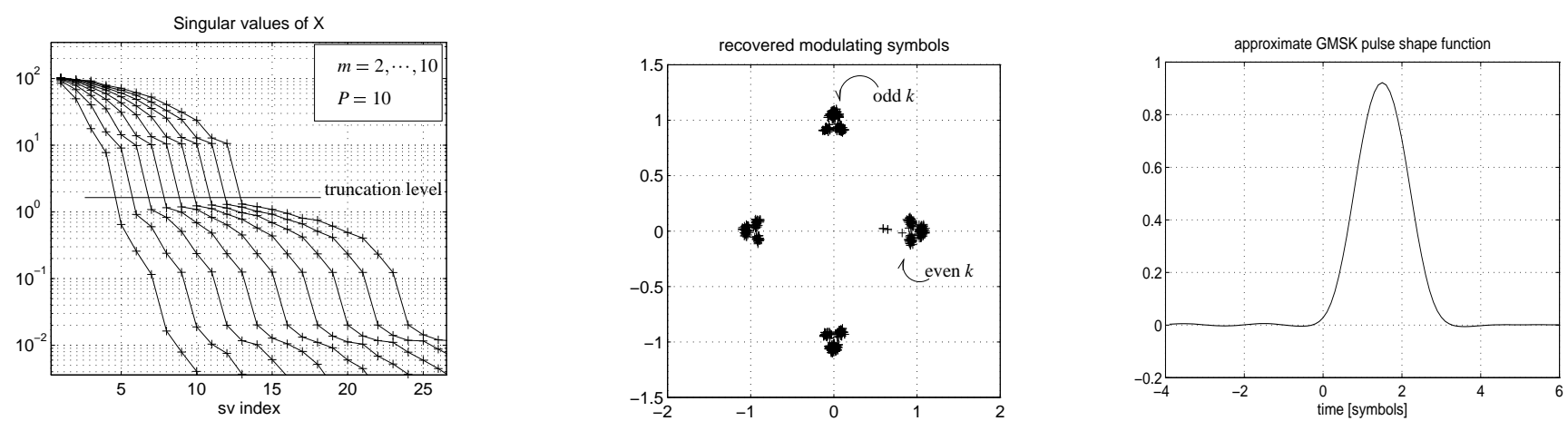

Figure 1. (a) Singular values of $\mathcal{X}$ for $m=2, \cdots, 10,(b)$ blindly identified modulating bits, $(c)$ estimated pulse shape function.

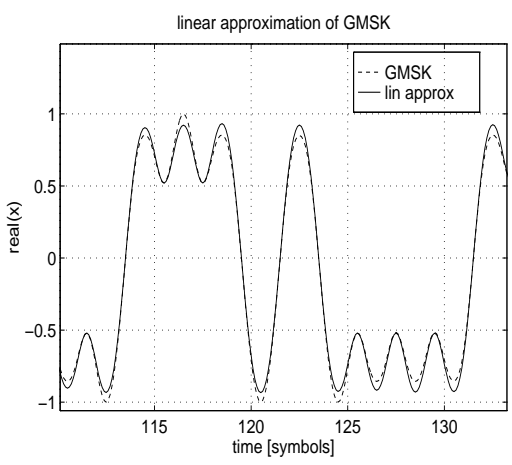

Figure 2. Real part of $x(t)$ and $\hat{x}(t)$.

\section{LINEAR APPROXIMATION OF A GMSK SIGNAL}

We first analyze GMSK signals in the absence of a channel. Thus let $x(t)$ be a GMSK signal with a normalized period $(T=1)$,

$$
x(t)=\exp j \phi(t), \quad \phi(t)=\sum_{k=0}^{N} d_{k} q(t-k),
$$

where the "phase impulse response" $q(t)$ is the integral of a gaussianshaped pulse whose precise form can be found in [7]. $q(t)$ is (approximately) zero for $t<-1.5$, (approximately) $\frac{\pi}{2}$ for $t>1.5$, and has a smooth transition in between. The modulating symbols $d_{k}$ are differentially encoded from the original data $s_{k} \in\{-1,1\}$ via $d_{k}=1$ if $s_{k}=s_{k-1}, d_{k}=-1$ if $s_{k}=-s_{k-1}$. Hence we obtain a $+\frac{\pi}{2}$ phase increase (smeared over 3 symbol periods) if there is no change in symbols in going from time $k-1$ to $k$, and $-\frac{\pi}{2}$ if there is a change. In this perspective, it is also relevant to define the MSK symbol constellation $\tilde{s}_{k} \in\{ \pm 1, \pm j\}$,

$$
\begin{cases}\tilde{s}_{k}=j \tilde{s}_{k-1} & \text { if } s_{k}=s_{k-1}, \\ \tilde{s}_{k}=-j \tilde{s}_{k-1} & \text { if } s_{k}=-s_{k-1}\end{cases}
$$

which would be the samples of $x(k)$ at integer sampling instants in case MSK modulation is used (i.e., $q(t)=\frac{\pi}{2} t, 0 \leq t \leq 1$ ).

From $x(t)$, construct $\mathcal{X}$ as in (1). The singular values of $\mathcal{X}$ for varying $m$ are plotted in figure $1(a)$. It is seen that, mathematically speaking, $\mathcal{X}$ is full rank, but in practice, it can be truncated at the indicated threshold since the gap is relatively large at that point. This leads to a reasonably accurate low-rank approximation of $\mathcal{X}$. E.g., for $m=4$, we obtain $\hat{d}_{\mathcal{X}} \approx 6$, so that the "channel length" $L_{p}$ is estimated from equation (2) to be $L_{p}=3$, as expected.

Blindly identifying the modulating symbols (i.e. $\mathcal{S}$ ) is straightforward since there is only one signal and the channel length is welldetermined. Using subspace intersections only, the algorithm in
$[2,3]$ produced symbol estimates that cluster around the MSK constellation points $\{ \pm 1, \pm j\}$, with phase changes of $\pm \frac{\pi}{2}$ : we have retrieved $\tilde{s}_{k}$ as defined in (3). See figure $1(b)$. From $\mathcal{X}$ and the $\tilde{s}_{k}$, we obtain that the corresponding modulating pulse shape function is as depicted in figure $1(c)$. The remodulated signal using this pulse and the MSK constellation gives a linearly modulated signal $\hat{x}(t)$ which matches $x(t)$ quite well, in particular in phase (figure 2).

Hence we see that the linear data model in section 2 is reasonably accurate for GMSK, despite its nonlinear (phase) modulation. For the purpose of blind identification, however, there is a catch. Because of the nonlinear modulation, other symbol constellations may occur, and we frequently obtain a constellation that is $\{ \pm 1 \pm j\}$, with phase changes of 0 or $\frac{\pi}{2}$ for even $k$, and 0 or $-\frac{\pi}{2}$ for odd $k$. The existence of a second constellation leads to some problems for blind identification algorithms that only force the Toeplitz structure and the FA constellation $\{ \pm 1, \pm j\}$, since it is not known beforehand which constellation we end up with, and the recovery of the original data $s_{k}$ depends on this. In section 5 a remedy is proposed, essentially consisting in forcing the constellation to be $\{ \pm 1\}$ on even $k$, $\{ \pm j\}$ on odd $k$.

\section{CHANNEL ANALYSIS BY SVD}

Now that we know that GMSK signals are well approximated by a linear model $\mathcal{X}=\mathcal{H} \mathcal{S}$, we analyze transmission over wireless channels. We use the ETSI-specified standard 12-tap "HT0" hilly terrain model [4], which has a channel length of $L_{c}=5$ symbol periods, so that the overall channel length (including ISI due to the GMSK modulation) is in the order of $L=7$ or 8. Figure 3(a) shows the singular values of $\mathcal{X}$, for varying $m$, and with $P=3$ times oversampling and $N=500$ samples. From the ranks of $\mathcal{X}$, the channel length is estimated as $L=3$ rather than 7 or 8: clearly, oversampling alone does not provide enough resolution to identify the channel length. The explanation is that the signal is bandlimited: the Nyquist rate is approximately equal to the symbol rate. As a consequence, the role of oversampling is limited: it does not make sense to have $P>2$ because the extra samples can be obtained by interpolation as well and do not introduce new information. Without sufficient resolution, perfect equalization is in general impossible. Nonetheless, the HT channel usually allows satisfactory equalization because the long-delay echos are rather weak.

Since GMSK signals do not have excess bandwidth, a result in [5] indicates that at least $M=2$ antennas are needed for detection of the channel length. Singular value plots for $M=2, P=3$ appear in figure $3(b)$. From the plots, the channel length is correctly estimated as $L=7$. For 2 users, [5] claims that 3 antennas are (barely) 

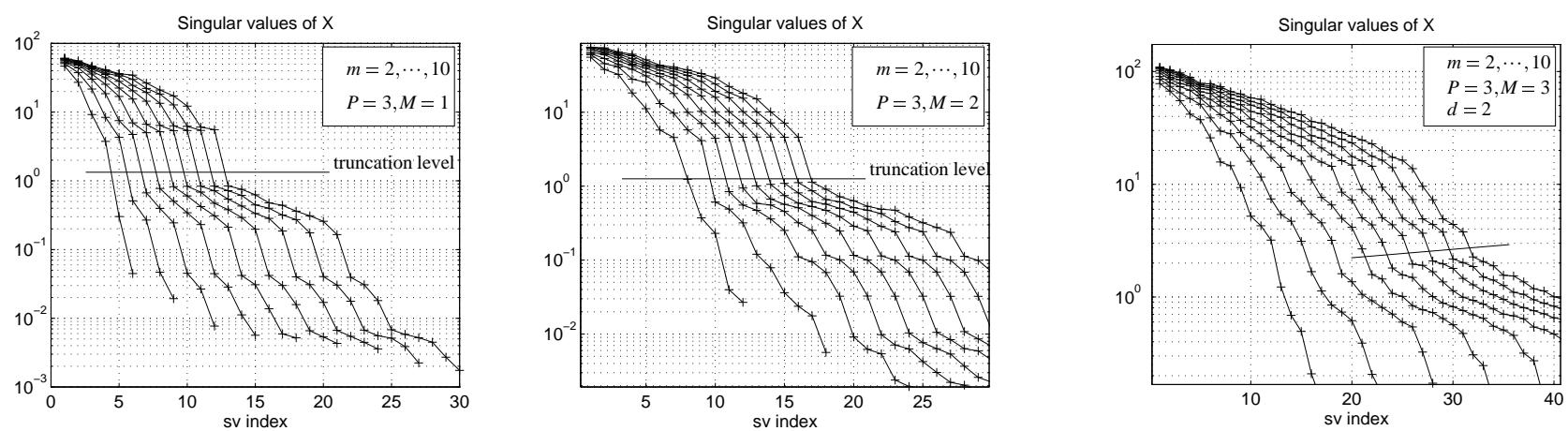

Figure 3. (a) Singular values of $\mathcal{X}$, HT channel, 1 antenna, (b) 2 antennas, (c) 2 co-channel signals with 3 antennas.

\section{BLIND CHANNEL IDENTIFICATION}

The blind symbol estimation algorithm in [3] is in principle suited for the equalization and separation of multiuser GMSK signals. It consists of two steps: (1) forcing the Toeplitz property of $\mathcal{S}$, by finding a basis for the row span of its generator $\left[\mathbf{s}_{-L+1} \cdots \mathbf{s}_{N-1}\right]$ (requiring the intersection of $L+m-1$ subspaces), (2) separating the signals using the FA property, by finding which independent linear combinations of the rows of the basis leads to sequences with entries belonging to the symbol constellation. So far, we have used the (suboptimal) iterative projection algorithm ILSP for that purpose [8]. The number of subspaces that are intersected can be reduced to $n, 1 \leq n \leq L+m-1$, which gives a larger basis from which ILSP has to form symbol sequences. Although theoretically this yields more accurate results (the intersection step with a large $n$ amplifies the noise), the performance of ILSP is often limited by the initialization of the iteration, which is harder for large bases and constellations larger than 2 points. A second problem, specific to GMSK signals, is that projection onto the symbol constellation $\{ \pm 1, \pm j\}$ does not always lead to the MSK constellation, requiring an additional noncoherent demodulation step. In this section, we propose solutions to both problems.

\subsection{Derotation}

The complex MSK constellation symbols $\left\{\tilde{s}_{k}\right\}$ in (3) can be transformed into the original real data symbols $\left\{s_{k}\right\} \in\{ \pm 1\}$ by a technique sometimes called "derotation":

$$
\begin{aligned}
& {\left[\begin{array}{ll}
\mathbf{s}_{0} & \mathbf{s}_{1} \cdots
\end{array}\right]=\left[\begin{array}{lll}
\tilde{\mathbf{s}}_{0} & (-j) \tilde{\mathbf{s}}_{1} & (-1) \tilde{\mathbf{s}}_{2} \cdots
\end{array}\right] D} \\
& =\left[\begin{array}{ll}
\tilde{\mathbf{s}}_{0} & \left.\tilde{\mathbf{s}}_{1} \cdots\right] D, \quad D:=\operatorname{diag}\left[(-j)^{k}\right.
\end{array}\right]_{k=0}^{N-1}
\end{aligned}
$$

Similarly, we can transform the Toeplitz matrix $\tilde{\mathcal{S}}$ constructed from $\tilde{\mathbf{s}}_{k}$ to $\mathcal{S}$ by premultiplying with a diagonal matrix $D_{\mathcal{H}}^{-1}$ as well:

$$
\mathcal{S}=D_{\mathcal{H}}^{-1} \tilde{\mathcal{S}} D, \quad D_{\mathcal{H}}^{-1}:=\operatorname{diag}\left[\begin{array}{llll}
1 & j & -1 & \cdots
\end{array}\right]=\operatorname{diag}\left[(j)^{k}\right]_{k=0}^{N-1} .
$$

Thus $\mathcal{X} D=\mathcal{H} \mathcal{S}$, where $\mathcal{H}=\tilde{\mathcal{H}} D_{\mathcal{H}}$. All entries of $\mathcal{S}$ are \pm , hence $\mathcal{S}$ is a real matrix. Consequently, we can work with

$$
\mathcal{X}_{R}=\left[\begin{array}{c}
\operatorname{re}(\mathcal{X} D) \\
\operatorname{im}(\mathcal{X} D)
\end{array}\right]=\left[\begin{array}{c}
\operatorname{re}(\mathcal{H}) \\
\operatorname{im}(\mathcal{H})
\end{array}\right] \mathcal{S}
$$

which is a data model in which all entries are real. Given $\mathcal{X}$, we form $\mathcal{X}_{R}$ and try to find the factorization (4). This brings us back to the problem of blind estimation of BPSK signals as considered in previous publications $[2,3]$. Since only the MSK constellation transforms to a real-valued $\mathcal{S}$, the constellation ambiguity has been resolved.

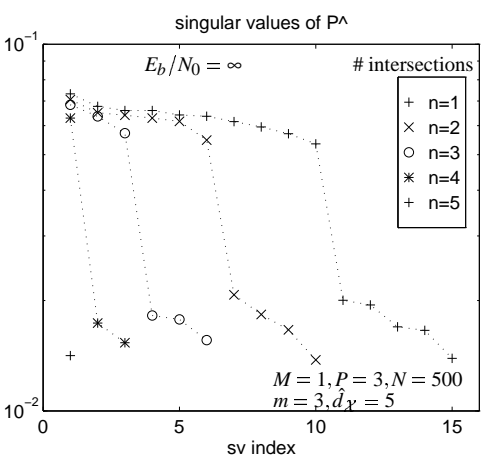

Figure 4. Singular values of $\hat{P}$, for $\mathcal{X}$ noisefree but truncated.

\subsection{Real ACMA}

To find accurate initial points for the ILSP algorithm, the constant modulus (CM) property of the signals can be used. This step fits in between the subspace intersection step, which is mainly used to reduce the dimensionality of the problem by $2 d n$ rows, and the FA step (ILSP), which is used for accurately converging to the symbols and the channel model. Although the CM step fits after the truncation of $\mathcal{X}$ to low rank and the intersection step, we will, for notational simplicity and without loss of generality, omit that step in the discussion in this section (i.e., we set the number of intersections to $n=1$ for the moment). The idea is to specialize the so-called "Analytic CMA" [6] to real signals. The main advantage of the ACMA in comparison with other CMAs is that it gives a non-iterative exact solution to the CM problem in the absence of noise, and that it is quite robust in finding all solutions, even with a substantial amount of noise added to $\mathcal{X}$.

The real-valued CM problem is to find all (real-valued) independent vectors $\mathbf{w}$ such that $\mathbf{w}^{T} \mathcal{X}_{R}$ is a sequence with all its entries equal to \pm 1 . Denoting the $i$-th column of $\mathcal{X}_{R}$ by $\mathbf{x}_{i}$, this is equivalent to

$$
\mathbf{w}^{T} \mathbf{x}_{i} \mathbf{x}_{i}^{T} \mathbf{w}=1, \quad i=0, \cdots, N-1 .
$$

Similar as in [6], these conditions can be rewritten in linear form by using Kronecker products: $\left[\mathbf{x}_{i} \otimes \mathbf{x}_{i}\right]^{T}[\mathbf{w} \otimes \mathbf{w}]=1$, but because $\mathbf{x}_{i}$ and $\mathbf{w}$ are real, it makes sense to remove duplicate entries in the Kronecker products. Thus define, for a $p \times p$ real symmetric matrix $Y=\left[y_{i j}\right]$, a stacking of the lower triangular part of the columns:

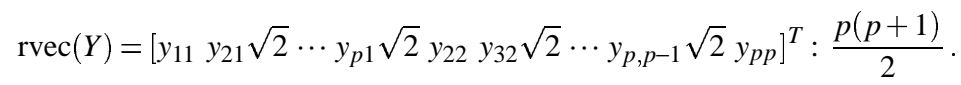

This allows to write (5) as 

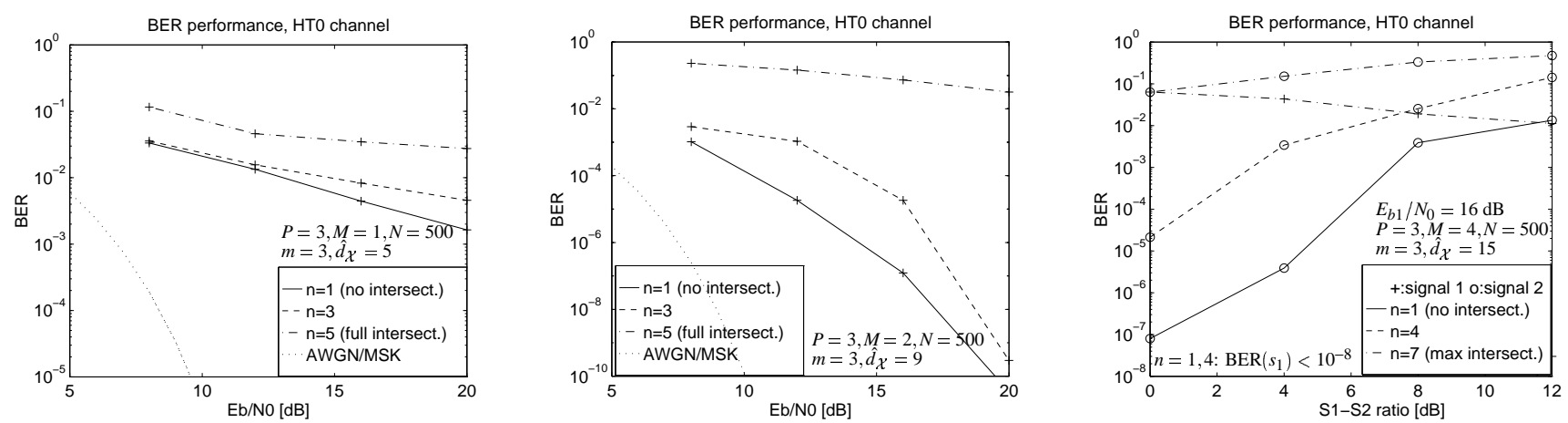

Figure 5. (a) Performance for varying number of intersections $n, 1$ antenna, (b) 2 antennas, $(c)$ separation of 2 signals using 4 antennas.

Collect all rows $\left[\operatorname{rvec}\left(\mathbf{x}_{i} \mathbf{x}_{i}^{T}\right)^{T}\right]$ into a matrix $P$. Then

$$
P \mathbf{y}=\mathbf{1}, \quad \mathbf{y}=\operatorname{rvec}\left(\mathbf{w} \mathbf{w}^{T}\right),
$$

where $\mathbf{1}=[1 \cdots 1]^{T}$. Hence, we have replaced the quadratic equations (5) by a linear system $P \mathbf{y}=\mathbf{1}$, subject to a quadratic constraint, which imposes a certain structure on $\mathbf{y}$. Similar as in [6], we can transform the linear system to

$$
\hat{P} \mathbf{y}=0, \quad\|\mathbf{y}\|>0
$$

of which the general solution is

$$
\mathbf{y}=\alpha_{1} \mathbf{y}_{1}+\cdots+\alpha_{\delta} \mathbf{y}_{\delta} \quad\left(\alpha_{i} \in \mathbb{R}\right)
$$

where $\left\{\mathbf{y}_{i}\right\}$ is a basis of the null space of $\hat{P}$, and $\delta$ is the dimension of this space. To force the structural property $\mathbf{y}=\operatorname{rvec}\left(\mathbf{w} \mathbf{w}^{T}\right)$, write $Y_{i}=\operatorname{rvec}^{-1}\left(\mathbf{y}_{i}\right)$, which gives

$$
\alpha_{1} Y_{1}+\cdots+\alpha_{\delta} Y_{\delta}=\mathbf{w w}^{T} .
$$

We have to find all parameter vectors $\left[\alpha_{1} \cdots \alpha_{\delta}\right]$ such that the linear combination of the matrices $\left\{Y_{i}\right\}$ is of rank 1 and symmetric, in which case it can be factored as $\mathbf{w} \mathbf{w}^{T}$. This is essentially a generalized eigenvalue problem. A technique for computing all $\alpha$-vectors is detailed in [6], for the general complex case, but a specialization to the present real case is immediate.

This RACMA algorithm appears to be quite reliable in finding estimates for all independent weight vectors $\mathbf{w}$. As in the complex case, the accuracy of the weight vectors is limited by the accuracy of the $\mathrm{CM}$ property, i.e., the amount of noise and the "structured noise" (or ISI) introduced by truncating the SVD of $\mathcal{X}$. Improved results are obtained by subsequently using $2-3$ iterations of the ILSP algorithm. The number of independent CM signals is indicated by $\delta$, the dimension of the null space of $\hat{P}$. Without long-delay multipath, this number is equal to $d$, the number of cochannel users; with multipath, the dominant echos add to $\delta$. Simulations on the HT channel model indicate that $\delta$ is equal to $\hat{d}_{\mathcal{X}}$, the estimated rank of $\mathcal{X}$. More generally, if $n$ subspace intersections are employed in the first step, then $\delta$ is reduced to $\hat{d}_{\mathcal{X}}-d(n-1)$ (figure 4).

\subsection{Simulations}

Figure 5(a) shows the BER performance of the resulting blind algorithm [derotation/truncation/intersection/RACMA/ILSP] for 1 signal and 1 antenna, with varying $E_{b} / N_{0}$, where $E_{b} / N_{0}:=\operatorname{SNR} \sqrt{P}$ is the "inband" signal-to-noise ratio. The simulation is based on 400 experiments of the 12-tap HTO channel [4], and included
Rayleigh fading, simulated by 40 local scatterers around the source. (The signal power was normalized in each experiment, so that the SNR is independent of the fading.) The graph shows that better results are obtained with less intersections, in which case the performance is comparable to that of a Viterbi equalizer [7, p. 732]. The performance saturates for large SNR, probably because of the resolution problem mentioned in section 4 . Significant improvements are obtained by the addition of a second antenna at $\lambda / 2$ (figure $5(b)$ ). The angles of arrival of the echos were set at $0^{\circ} \pm 2^{\circ}$ for the short-delay echos, and $100^{\circ} \pm 40^{\circ}$ for the large-delay $(>15 \mu \mathrm{s})$ echos. (For small BERs, the BER is computed from the variance of the estimates.)

Figure 5(c) shows the performance for 2 co-channel users and 4 antennas. The second user has a similar (slightly modified) HT channel. Angle offsets were, for signal 1 : $\left[0^{\circ} \pm 3^{\circ} ; 80^{\circ} \pm 20^{\circ}\right]$; for signal 2: $\left[40^{\circ} \pm 3^{\circ} ;-40^{\circ} \pm 20^{\circ}\right]$. The SNR was set at $E_{b} / N_{0}=16 \mathrm{~dB}$ for signal 1 , and the power of signal 2 was varying between $0-12 \mathrm{~dB}$ below signal 1 . With a reduced number of intersections, signal 1 is recovered virtually error-free, and signal 2 is found quite reliably as well, with a performance comparable to that of the single-user scenario with 2 antennas.

\section{REFERENCES}

[1] H. Liu and G. Xu, "A deterministic approach to blind symbol estimation," IEEE Signal Processing Letters, vol. 1, pp. 205207, Dec. 1994.

[2] A.J. van der Veen, S. Talwar, and A. Paulraj, "Blind estimation of multiple digital signals transmitted over FIR channels," IEEE Signal Processing Letters, vol. 2, pp. 99-102, May 1995.

[3] A.J. van der Veen, S. Talwar, and A. Paulraj, "Blind estimation of multiple digital signals transmitted over multipath channels," in Proc. IEEE MILCOM, (San Diego), Nov. 1995.

[4] European Telecommunications Standards Institute, "European digital cellular telecommunications system (phase 2): Radio transmission and reception (GSM 05.05)," tech. rep., ETSI, Sophia Antipolis, France, 1994.

[5] A.J. van der Veen, "Resolution limits of blind multi-user multichannel identification schemes - the bandlimited case," in Proc. IEEE ICASSP, (Atlanta, GA), May 1996.

[6] A.J. van der Veen and A. Paulraj, "An analytical constant modulus algorithm,” IEEE Trans. Signal Proc., May 1996.

[7] R. Steele, ed., Mobile Radio Communications. IEEE, 1994.

[8] S. Talwar, M. Viberg, and A. Paulraj, "Blind estimation of synchronous co-channel digital signals using an antenna array. Part I: Algorithms,” IEEE Trans. Signal Proc., May 1996. 